\title{
LA INSTITUCIÓN ESCOLAR: \\ ¿ENCUADRE O DESACOPLE EN LA FORMACIÓN PERMANENTE DOCENTE? UNA APROXIMACIÓN A LAS CONCEPCIONES DOCENTES.
}

Irene Marina Macera (UNR-CONICET)*

\section{Resumen}

En esta presentación identificamos las concepciones docentes acerca de la formación permanente contextualizando las mismas en el ámbito escolar y social en el cual se desarrolla la vida cotidiana docente. Distinguimos, para ello, dos situaciones institucionales que impregnan de sentidos las concepciones acerca de la formación: una escuela pública y una escuela privada, ambas de la ciudad de Rosario. Cabe aclarar que aquello que interesa de esta distinción es poder pensar en la pertenencia institucional, las relaciones entre colegas y con directivos, los espacios institucionales de encuentro, los canales comunicacionales, la participación docente en la dinámica institucional, es decir, todas aquellas actividades por fuera del aula, que intervienen en la construcción de las concepciones docentes y de su trabajo cotidiano.

En otras palabras, los modos de utilización y distribución de recursos institucionales, de circulación y socialización de ideas, de acción y participación que tienen los docentes en cada una de las instituciones en que desempeñan su tarea, crean modos diferenciales de concebir la formación docente.

En este sentido, nos proponemos relacionar las características institucionales y las concepciones docentes respecto de la formación permanente.

\section{Palabras claves}

Políticas educativas - Formación permanente - Institución escolar - Cotidianeidad Concepciones docentes.

\footnotetext{
Abstract

In this presentation we identify the teachers' conceptions about permanent formation contextualized in the social and educational field in which teachers' daily

Licenciada y Profesora en Ciencias de la Educación. Doctoranda en Humanidades y Artes con mención en Ciencias de la Educación, Facultad de Humanidades y Artes, Universidad Nacional de Rosario. Becaria Tipo II del CONICET.
} 
life take place. We distinguish two different institutional situations that give meaning to the conceptions of permanent formation: a public school and a private school, both in the city of Rosario. This distinction is interesting and helpful to think about institutional membership, relations between colleagues and authorities, institutional meeting spaces, the communication channels, teachers' participation in the institutional dynamics, and all those activities outside the classroom, involved in building theirs' educational conceptions and theirs' daily work.

In other words, we are interested in the modes of operation and distribution of institutional resources, in the circulation and socialization of ideas, in the action and involvement of teachers in each institution where they work and where they create differential modes of thinking about theirs' permanent formation.

In this sense, we propose to connect the institutional characteristics and the teachers' conceptions of permanent formation.

\section{Key Words}

Educational policies - Permanent formation - Educational institution - Daily life Teachers' conceptions.

\section{Acerca del proceso de investigación}

El proyecto de investigación en curso, se ocupa de la indagación y análisis interpretativo de las concepciones docentes acerca de su trabajo y formación en el contexto de las políticas educativas de la década de los '90 hasta la nueva Ley de Educación Nacional, 2006. La investigación se titula "Políticas educativas y transformaciones en el trabajo y formación docente. Un análisis de las concepciones docentes acerca del trabajo y los nuevos requerimientos de formación" y se enmarca en una beca doctoral de Conicet y participa del Centro de Estudios Antropológicos en Contextos Urbanos (CEACU).

Una de las dimensiones del proceso de investigación, supone el trabajo con los documentos que definen los nuevos rumbos educativos, las concepciones docentes acerca de los mismos y las concepciones docentes acerca de su implementación, en tanto que ambos responden a la vida cotidiana de las escuelas. La norma, en su letra e implementación, interviene directamente en lo cotidiano. Es el estudio de las concepciones docentes desde donde hicimos foco para poder pensar el trabajo y la formación docente de nivel medio de la ciudad de Rosario.

Nos propusimos la construcción del contexto político educativo nacional y las posiciones que toma la provincia de Santa Fe con respecto al mismo en el período de la Ley Federal de Educación (1993) y la Ley de Educación Nacional (2006) a través de las leyes y resoluciones que les dan concreción, al mismo tiempo que iniciábamos el proceso de entrevistas abiertas y en profundidad con docentes provenientes de distintas áreas disciplinarias, distintas instituciones escolares y distintos años de antigüedad en la profesión. 
Nuestro objetivo fue trazar una suerte de mapa de concepciones docentes que diera cuenta de su diversidad y/o similitudes y/o puntos de contacto, a partir de sus experiencias de trabajo en relación con los imperativos que se sucedían en las políticas educativas.

Así como buscamos que la producción de conocimiento de nuestra investigación de cuenta de las relaciones entre las concepciones docentes que se elaboran en los cotidianos escolares y las políticas educativas, el proceso metodológico es diseñado y desplegado, también, en esta dirección: un "proceso recursivo" (Achilli, E.L., 2005), "circular” (Mendizábal, N. 2007) entre el análisis interpretativo documental, el análisis interpretativo de entrevistas docentes, la recopilación de los antecedentes de investigación y la teoría.

Dicho posicionamiento de la investigación supone un "diseño de investigación flexible" que se define por estar disponible a modificar preguntas, propósitos y/o estrategias metodológicas de la investigación en función de las situaciones inesperadas que pueden presentarse en el transcurso del proceso metodológico, una producción de datos descriptivos, enriquecidos por la presencia de la palabra de los entrevistados, en donde se busca captar reflexivamente el significado de la acción atendiendo a la perspectiva del sujeto y el abordaje holístico de las situaciones sociales complejas (Mendizábal, N. 2007; pp. 68-69).

El proceso de investigación asume ciertas categorías para pensar el trabajo y la formación permanente docente desde la perspectiva de las concepciones docentes, vida cotidiana y concepciones desde un enfoque relacional. El enfoque relacional supone el tratamiento de tres núcleos problemáticos: acceder al conocimiento de las situaciones que se viven cotidianamente ya sea como rutina o como singularidad imperceptible de rupturas; los sujetos sociales; la dialéctica entre trabajo de campo y trabajo conceptual (Achilli, E.L, 2005). Se trata de conocer los procesos en el contexto histórico en que se inscriben, tener presente el carácter de movimiento de las prácticas y relaciones sociales, su estructura hegemónica y los distintos niveles de conflictividad (Achilli, E.L, 2005).

Es en el siglo XIX donde el estudio de la vida de los hombres en sociedad, la experiencia de la gente común en la vida cotidiana, el interés por descubrir el vínculo entre la biografía individual y los procesos sociales amplios -dirá Bauman-, define a la sociología y se constituye por este modo de explicar la condición humana a través del análisis de múltiples redes de interdependencia humana (Bauman, Z., 1994). Referentes destacados, continuadores del pensamiento fenomenológico del filósofo Edmund Husserl, son Alfred Schutz, Peter Berger y Tomas Luckman cuyas obras importantes acerca de la vida cotidiana datan desde mediados del siglo XX.

También es la filosofía la que comienza a interrogarse por la pertinencia del estudio de la vida cotidiana y el sentido común hacia el siglo XIX. Destacamos filósofos provenientes de la corriente marxista, como lo son Agnes Heller y Henri Lefebvre cuyas obras de lo cotidiano se publican hacia fines de los 
años sesenta y setenta, a Guy Debord, filósofo y cineasta francés, referente del Situacionismo que a partir de la amistad que engendra con Henri Lefebvre ha producido pensamiento acerca de lo cotidiano y, por último, un filósofo e historiador que también ha escrito las décadas del 60 y 70 francés, que consideramos un hallazgo para pensar ciertas prácticas y concepciones de la vida cotidiana docente, Michel De Certeau.

En el recorrido teórico sobre la noción de concepción, advertimos que toda concepción del mundo se construye y desenlaza en la experiencia y el testimonio de esa experiencia, por tanto, consideramos que el testimonio es territorio de las concepciones. En el testimonio hay un sujeto presente que posee la cualidad de transformarse, es la palabra viva de un sujeto que relata, narra una experiencia situada, dotando de sentido un tiempo y un espacio pero que también esconde, sin saberlo, restos de experiencia. Sentidos, sinsentidos, significaciones, signos, sentido común, filosofía, emergen en el testimonio y se anudan en la construcción de una "concepción de mundo". En la noción de concepción, tal como la entendemos, están presentes, entonces, lo dicho, no-dicho, lo contradictorio, junto con el pensamiento y la opinión. Polifonía de elementos, voces, texturas, experiencias, repeticiones dan origen a la noción de concepción.

Hablar de concepciones, entonces, nos permite aproximarnos hacia aquellos elementos sociales y culturales que actúan en la construcción de ideas, imágenes, funciones, y/o ideales, que realiza el sujeto docente para definir (se) su trabajo y formación. Da cuenta de la polifonía de voces e interlocutores que están actuando en esta construcción cotidiana de sus concepciones.

El trabajo de campo realizado supone entrevistas individuales y colectivas con docentes del nivel medio de la ciudad de Rosario, de diferentes instituciones educativas y diferentes años de antigüedad en la docencia.

Desde el inicio, nos propusimos contactar a los docentes desde contactos informales y no desde las instituciones donde desarrollan su trabajo. Este fue un primer criterio para acceder sus concepciones. Otro criterio fue, como adelantamos, la realización de entrevistas abiertas y en profundidad en torno a la problemática de su trabajo y formación, en donde cada entrevista auspicie como el territorio a través del cual se desprendan los núcleos problemáticos para profundizar en una próxima instancia y, del devenir mismo del proceso metodológico de investigación. De esta deriva metodológica emerge la idea de lo que dimos en llamar "espacio de indagación colectiva", encuentros colectivos donde trabajamos núcleos comunes de conversación extraídos de las entrevistas individuales que sirven para poner en tensión las concepciones docentes posibilitando una mayor claridad, densidad y complejidad de las mismas. 


\section{El contexto de la formación permanente docente}

En 1993, se sanciona la Ley Federal de Educación № 24.195 (LFE) que define la educación como bien social y responsabilidad común y señala el inicio de la reconversión del sistema nacional de educación necesario para adecuarse a las nuevas necesidades nacionales (LFE, Art. $1^{\circ}$ ). En el Art. $66^{\circ}$ se hace mención a la implementación de programas de formación y actualización para la docencia para adaptarse a las necesidades de la nueva estructura (LFE, Inc. D) de los nuevos niveles de Educación Inicial, Educación General Básica y Polimodal.

El derecho y deber del docente se estructura en la capacitación, actualización y nueva formulación en servicio para adaptarse a los cambios curriculares requeridos (LFE, Art. $46^{\circ}$ Inc. I. /Art. $47^{\circ}$. Inc. D, respectivamente) y en afianzar el sentido de la responsabilidad en el ejercicio de la docencia. La educación, de aquí en más, es entendida como proceso permanente (LFE, Art. $5^{\circ}$ Inc. 9).

Los objetivos de la formación docente que se promulgan en dicha ley son: preparar y capacitar para un eficaz desempeño en cada uno de los niveles y modalidades (LFE, Art. 19. Inc. A); perfeccionar con criterio permanente a graduados y docentes en actividad en los aspectos científico, metodológico, artístico y cultural. Formar investigadores y administradores educativos (LFE, Art. 19. Inc. B); formar al docente como elemento activo de participación en el sistema democrático (LFE, Art. 19. Inc. C); fomentar el sentido responsable de ejercicio de la docencia y el respeto por la tarea educadora (LFE, Art. 19. Inc. D).

En junio de 1994, la Resolución 36/94 aprueba el Documento Serie A No 9 "Red de Federal de Formación Docente Continua" (RFFDC) definida como un sistema articulado de instituciones que aseguren la circulación de la información para concretar las políticas nacionales de formación docente continua, acordadas en el ámbito del Consejo Federal de Cultura y Educación (...) Su finalidad será ofrecer un marco organizativo que facilite las articulaciones intra e interprovinciales para el desarrollo de un Plan Federal de Formación Docente Continua.

En 1995, en base a la LFE se sanciona la Ley de Educación Superior N ${ }^{\circ}$ 24.521 (LES). Se manifiesta como objetivo de la misma incrementar y diversificar las oportunidades de actualización, perfeccionamiento y reconversión para los integrantes del sistema y sus egresados (LES, Art. $3^{\circ}$ Inc. I). Aquí vuelven a manifestarse los derechos y deberes de los docentes centralizados en la actualización y perfeccionamiento de modo continuo (LES, Art. $11^{\circ} \mathrm{Inc}$. C), actualizarse en su formación profesional y perfeccionarse en su carrera académica (LES, Art. $12^{\circ}$ Inc. C), participar en la vida institucional y cumplir su función de docente, de investigación y de servicios (LES, Art. $12^{\circ}$ Inc. B). En septiembre del 1996 se aprueban las "Bases para la organización de la formación docente" en la resolución 52/96. Se manifiestan las cuatro instancias de la formación docente continua: 
a) la 'formación de grado'; b) el 'perfeccionamiento docente en actividad'; c) la 'capacitación de graduados docentes para nuevos roles profesionales'; d) la 'capacitación pedagógica de graduados no docentes' (...) Las provincias, la Ciudad de Buenos Aires y las instituciones universitarias podrán organizar actividades correspondientes a las instancias b), c) y d) de la formación docente continua en las instituciones de formación docente y/o en instituciones específicamente organizadas para tal fin acreditadas en la Red Federal de Formación Docente Continua.(Resolución 52/96, CFCyE)

En octubre de 1997, la Resolución 63/97 aprueba el documento "Transformación Gradual y Progresiva de la Formación Docente Continua" donde se define la formación docente inicial para el desarrollo de competencias para el ejercicio profesional docente. Es así como la capacitación, el Perfeccionamiento y la actualización se orientan, según esta resolución, al "mejoramiento de la educación y a la profundización de los niveles de profesionalización de los docentes" (Resolución 63/97, Ministerio de Educación y Cultura de la Nación). En junio del año 2000 a partir de la Resolución 135/00 se comienza a hablar de la necesidad de elaborar el Pacto Federal Educativo II. Hacia mediados del año 2000 se establecen las "Bases para el Pacto Federal Educativo II" entre los diputados nacionales de la alianza y el Ministerio de Educación de la Nación. En el mismo se habla de la "jerarquización de la profesión docente" y se establece que los postítulos y posgrados sean de carácter voluntario. Aparece manifiesta la idea de concentrar las horas de los profesores en una sola institución, de mejorar los salarios al personal de aquellas escuelas que demuestren mejorar su desempeño y que se dejara constancia de dicha mejora en la historia laboral de cada docente. Se habla de incremento salarial a aquellos que se estén formando en posgrados y postítulos y/o que se comprometan con la institución en la que se desempeñan. En noviembre del año 2000, la Resolución 144/00, se ocupa de establecer una norma general para los postítulos docentes. En el anexo de dicha resolución se afirma que los objetivos de los postítulos docentes son:

La actualización y especialización dentro de un campo disciplinar o de problemáticas específicas de la formación docente; La profundización de nuevas experticias que permitan fortalecer la capacidad del docente en su práctica profesional y la profundización de la formación en investigación educativa. (Resolución 144/00)

En el mes de diciembre del 2000, la Resolución 152/00 se ocupa de la jerarquización docente y aprueba una comisión para estudiar y analizar los criterios básicos comunes para establecer la misma. En mayo del 2001, según Resolución 167/01, se resuelve poner en discusión los criterios básicos comunes que estableció la Comisión Especial para la jerarquización docente con las organizaciones gremiales. El documento que se presentó para la dis- 
cusión se llama "Análisis y Estudio de los Criterios Básicos Comunes para la Jerarquización de la Profesión Docente".

De mayo del 2001 a febrero del 2002 no se registran resoluciones. La primera resolución del año 2002 hace referencia al estado de emergencia edilicia en la que se encuentran una cantidad de establecimientos escolares poniendo en riesgo el comienzo de clases de ese año. En Abril del 2002, la Resolución 173/02 aprueba la puesta en marcha del Plan Federal Educativo.

En mayo del año 2003 asume la presidencia Néstor Kirchner. El Ministro de Educación que ocupa el cargo en todo el mandato presidencial es Daniel Filmus. Entre las transformaciones acerca del trabajo y la formación docente que requiere la Ley Federal de Educación (1993) -junto con la serie numerosa de documentos y resoluciones ministeriales que le dan concreción- y la nueva gestión de gobierno que se inaugura en el 2003, materializada en la Ley de Educación Nacional en el año 2006, encontramos un nuevo rumbo político de la educación.

Consideramos que presenciamos dos décadas que han pensado e implementado de modos muy diferentes las acciones educativas y los modos de entender a los distintos actores educativos. Esto puede constatarse en el Informe Final de la Comisión Federal de Formación Inicial y Continua elaborado en diciembre del 2005 en donde se expresa un diagnóstico muy crítico de las políticas de formación docente inicial y continua, promulgados e implementados en la década anterior, y con fuertes incidencias hasta esos momentos. Algunas de las críticas que queremos destacar en este documento son: la profundización del fenómeno del credencialismo en la formación; la configuración de un Estado Evaluador en lugar de un Estado rector del desarrollo del sistema formador; la oferta de capacitación privada desordenada e incierta, la crisis del sentido de lo público, de los valores éticos y sociales de la educación como factor de movilidad social; el cuestionamiento a la escuela como institución relevante para la socialización de las jóvenes generaciones; la culpabilización hacia los docentes por la baja competitividad de la escuela frente a las exigencias de la globalización y de un mercado de trabajo cada vez más excluyente; la profundización de la fragmentación del sistema educativo.

\section{La formación permanente en las instituciones (1)}

Para abordar las relaciones entre formación permanente, trabajo e institución, es preciso abordar esta última en la vida cotidiana docente. ¿Qué influencias tiene la institución en la formación permanente de nivel medio? ¿Qué lugar ocupa lo institucional en la formación permanente de nivel medio?

En primer lugar, podemos decir que la institución escolar es el lugar donde transcurre el trabajo específico del docente. La condición de ser profesor de una disciplina exige "tomar horas" en distintas instituciones escolares. Abundan los casos donde las docentes tienen desparramadas sus horas por tres, cuatro 0 
cinco escuelas. El lugar escuela, en el trabajo cotidiano de nivel medio docente, estaría sujeto a las experiencias pedagógicas, institucionales y laborales que allí acontecieran. Son éstas las que construyen, deconstruyen o destruyen un espacio, un lugar subjetivo, una escuela.

"La realidad es que los docentes de media trabajamos en dos, tres, cinco escuelas. ¡¿Qué involucramiento podes tener con la institución cuando andas corriendo de una escuela para la otra?!" (Registro $N^{\circ} 2$. Entrevista con "B". 18 de Octubre del 2007).

"Es tan difícil poder pensar otra escuela!. Aunque poder pensarla, por ahí...pero poder actuarla (...) No tenemos con quién pensar la educación que queremos" (Registro № 1. Entrevista colectiva. 09 de Agosto del 2008).

Las dificultades para involucrarse, para encontrar en la escuela interlocutores con quienes pensarla y pensar "otra" escuela, nos remite a la noción de no-lugar elaborada por Augé (2001). El no-lugar se define como espacio de circulación, comunicación y consumo, no hay modo de expresar la identidad, la historia y la relación. A diferencia del lugar que se define por la inscripción de marcas objetivas de identidad, de relación y de historia y por los modos de relación con los otros que prevalecen allí. Sin embargo, afirma este autor, las fronteras entre lugar y no-lugar se han desplazado y los lugares pasan a definirse en función de lo que ocurre allí, de su capacidad para producir subjetivación. El no-lugar que trabaja Augé, es física y materialmente identificable -aeropuertos, avenidas, pantalla de televisión- pero también se define como un espacio creado por una mirada que lo toma por objeto y, en este sentido, el no-lugar de unos puede ser el lugar de otros. Cuando se crea un lugar en un no-lugar se trata de un lugar subjetivo y de los vínculos simbólicos que se manifiestan en el espacio concreto del no-lugar (Auge, M., 2001).

El desplazamiento de fronteras entre lugar y no-lugar, se refiere a la transformación del sentido de lo público. Si el espacio público, que antes se identificaba con lugares específicos donde se trataban los asuntos públicos, es tomado por la pantalla de televisión -materialmente definido como nolugar- puede afirmarse que el espacio público, en general, y de la escuela, en particular, es a construir, no está dado. La escuela podría convertirse en no-lugar y, el aula, no obstante, podría sostenerse como lugar. Pero en ambos casos, hablaríamos de lugares y no-lugares subjetivos, esto es, que pueden serlo para unos y no para otros.

El sentido de lo institucional se dispersa por las múltiples subjetividades que lo habitan. Esta dispersión nos habla de la fragilidad de los sentidos comunes sociales contemporáneos, al mismo tiempo que empuja a la construcción de sentidos institucionales más situacionales, que den cuenta de las prácticas y relaciones cotidianas que allí se construyen. 
Desde esta mirada, comprender las relaciones entre formación permanente e institución desde las concepciones docentes, nos conduce a acercarnos a la cotidianeidad de una institución escolar, asumiendo particularidades, singularidades que no necesariamente puedan hallarse en otras experiencias institucionales así como también recurrencias que, cual sedimentaciones del pasado, se repiten naturalmente en lo institucional.

A través de las entrevistas individuales y colectivas, creamos una cartografía de cómo son las instituciones escolares donde trabajan las docentes y qué ocurre en ellas con respecto a la formación.

Una de las experiencias escolares docentes que elegimos para trabajar aquí, se vincula a una escuela pública, con gran matrícula de estudiantes, ubicada en el macrocentro de la ciudad de Rosario. Conocida por ser una escuela donde asisten chicos repetidores o de barrios alejados del centro y donde están muy presente las diferencias entre el turno mañana y el turno tarde relacionadas con el nivel cultural de los chicos.

La planta docente es muy numerosa, alumnos y profesores circulan bastante anónimamente. Hubo muchas directoras durante el transcurso de la trayectoria laboral de las docentes entrevistadas que trabajan allí. El edificio es el clásico edificio de escuela tradicional y de época, muy amplio e imponente, mantenido en gran parte por los fondos de la cooperadora de la escuela. Las aulas, dada la amplitud, se transforman en verdaderos mundos escolares, acompañadas de pasillos anchos y escaleras.

"además de ser muy grande, la institución en sí no es contenedora, hace agua. (...) Lo que me favorece es que nadie viene a controlarte, yo cierro la puerta y hago lo que quiero (...) la escuela te deja hacer, para bien o para mal (...) no te ofrece resistencias" (Registro $\mathrm{N}^{\circ}$ 1. Entrevista con G. 16 de mayo del 2007).

"la institución está ausente, yo lo que quiero instalar en la escuela es el debate porque estamos en la escuela muy adormecidos" (Registro $\mathrm{N}^{\circ} 2$. Entrevista con G. 11 de octubre del 2007).

Así lo describe una de las docentes que desempeña su tarea en esta institución. En este caso, se trata de una docente con todas sus horas titulares allí, de más de veinte años de antigüedad en la docencia. Sin embargo, no es la institución la que contiene su trabajo cotidiano. Las relaciones de empatía que allí se establecen, o quedan en el aula o se desarrollan afuera de la escuela. Con respecto a la formación permanente, afirma:

"...el grupo de matemáticas vamos en bloque. Funcionamos bastante bien, nos ponemos de acuerdo qué bibliografía usamos o, discutimos por qué no. Y nos pasamos material, es perfeccionamiento entre nosotras, es juntarte con el otro a ver qué hizo, eso es muy interesante. Por lo general todo esto que te cuento o lo hacemos 
extra clase o en los diez minutos de recreo obviamente que si tenemos que ver bibliografía nos vamos a otro lugar pero la escuela no te da ese espacio hemos formado un lindo grupo matemática y física (Registro No 1. Entrevista con G. 16 de mayo del 2007)."

Este grupo de docentes, produce espacios de autoformación en bares o casas y, al mismo tiempo, legitima el trabajo de cada una fuera de la escuela y dentro del aula, en tanto no se establecen espacios institucionales que posibiliten este tipo de encuentros.

En los primeros años de implementación de la formación permanente, a partir de la Ley Federal de Educación, las docentes asistieron a numerosos cursos. Luego, los mismos se privatizaron y, aún así siguieron recurriendo a estos espacios. En ambos casos, la formación permanente se presentó como un acto individual y un gesto de los propios docentes: intercambiar y socializar la formación de cada una para trabajar en el aula.

Observamos, entonces, la dimensión colectiva como reconocimiento y valoración del trabajo del cual la formación permanente es parte. Una dimensión colectiva autogestionada que recurre a experiencias subjetivas, las experiencias entre docentes, para hacerse un lugar, para dar sentido al trabajo cotidiano. La formación permanente, en otras palabras, surge en un entramado de relaciones docentes que no solo la hacen posible sino, y sobretodo, le otorgan significación en el contexto de trabajo áulico cotidiano.

En circunstancias donde no hay espacios institucionales, las exigencias de formación que plantea la Ley Federal de Educación devienen en "proletarización" de su tarea.

"empezaron estos cursos y fue como una maratón de cursos por otra parte para otros era negocio la maratón de cursos" (Registro $N^{\circ}$ 1. Entrevista con B. 13 de mayo del 2007)

"los cursos son para juntar puntos. Los puntos sirven para estar más arriba en el escalafón. La gente que ya tiene las horas hace otro tipo de posgrados, todos estos cursos de la red federal, no son por convicción ni por nada sino por puntos (...) Eh, yo la verdad que los cursos los hice por los papeles y después me enganche a hacerlos porque era tratar de entender lo que baja el estado en cuanto a cuáles son los paradigmas para enseñar las ciencias sociales en el neoliberalismo. A mí por ahí lo que me cambio o tuvo un impacto en mi práctica fue un curso de Indeso y lo de la maestría. Tienen que ver con aprender a otro nivel las cosas que pasan en las escuelas. Esta cosa del poder entre los grandes, entre los chicos (...) a partir de la ley federal hay un exceso de cosas. Muchas reuniones, nos hacen hacer muchas planificaciones y proyectos que después no lee nadie. Yo creo que es una práctica de control, un disciplinamien- 
to de cabeza: hagan esto por más que no sirva para nada" (Registro № 2. Entrevista con B. 18 de octubre del 2007).

Los enunciados que siguen a continuación son de docentes que se desempeñan en una institución privada de la ciudad de Rosario de quince años de antigüedad situada en barrio norte. El ingreso a la escuela es por un portón que da a un parque en el que están dispersos distintos edificios modernos y algunos antiguos reciclados. En ellos, se dan clases, hay una biblioteca -con una cantidad de computadoras considerable-, la sala de profesores, las oficinas de los directivos, de los coordinadores docentes y los tutores, entre otras cosas.

En el recreo, todo el espacio verde está dispuesto para el ocio, además de salones donde se transmite una radio abierta por los alumnos de quinto año.

"esta escuela es producto del proyecto menemista y así como hizo agua ese proyecto, esta escuela también. Entonces los papás de esta escuela que creían, igual que los docentes, en el proyecto decidimos que si ellos vienen a trabajar ad honorem nosotros cobramos discontinuo, con varios meses de discontinuidad, para tratar de salvar a la escuela. Eso nos pusimos como meta (...) los padres hacen de todo, están levantando toda la escuela porque vienen acá, van al ministerio, golpean puertas, hacen trámites judiciales, invierten su tiempo, porque no cobran nada, incluso hay abogados y contadores, ponen todo lo que saben (...) y nosotros somos los responsables de llevar adelante el proyecto" (Registro N ${ }^{\circ} 1$. Entrevista con P. 08 de junio del 2007).

La historia institucional de esta escuela ha incluido a docentes, directivos, padres y la comunidad educativa en general para hacer frente a la supervivencia de la misma. Está presente una apuesta propia y colectiva, por parte de todos estos actores fundacionales acerca de un proyecto común de educación. Así con el transcurso de los años, van agregando más cosas, actividades extraescolares para los chicos, más espacios institucionales para los docentes. Sobre este último punto, veamos los siguientes testimonios.

"Lo que nosotros tenemos es nuestro propio perfeccionamiento interno, tenemos una licenciada en psicopedagogía que trabaja todo el año con nosotras, plantea dificultades y va haciendo reuniones progresivas hasta que llegamos a la conclusión. Ella tiene un taller que se llama Aprender a Aprender y trabaja con alumnos y con docentes. Con los docentes va trabajando el problema específico de aprender y te va haciendo ver otras estrategias que uno puede usar. Ella está disponible en la escuela, le decís tengo este problema puntual con este alumno entonces ella te alcanza material para leer, te sugiere. Es una persona que está al alcance, es muy capaz. Te presenta todo y te dice qué hacemos con esto y ahí discutimos en- 
tre todos, entonces, es un aprender interesante (...) La supervisión te acompaña porque esto es un proyecto. Nosotros no trabajamos independientemente del ministerio sino que tenemos un proyecto interno de la escuela que se preocupa mucho por la formación docente (...) A nivel formal, eso de que te piden créditos, papeles no hay. El perfeccionamiento docente viene como implícito pero no es una exigencia formal, no te preguntan (en la escuela) cuántos cursos hiciste este año y eso va a tu evaluación. No está instalada esta exigencia al docente, en la escuela pública, cero y en la escuela privada no es un requisito fundamental. Acá hay gente que por ahí en todo el año no hace nada" (Registro $N^{\circ} 1$. Entrevista con S. 10 de diciembre del 2009)

En estos enunciados nos encontramos con espacios institucionales de formación abocados a las problemáticas de aprendizajes, de enseñanzas, relaciones intergeneracionales 0 , acerca de las inquietudes que surgen entre los actores educativos que trabajan en la institución. En las reuniones con la psicopedagoga -referidas en la cita- también asisten los profesores talleristas que trabajan con los alumnos en contraturno, promoviendo un espacio de escucha y de valoración de saberes y prácticas diversas. Aún cuando estas horas institucionales de formación no se contemplan en el salario docente, puede observarse, la importancia que le otorgan las docentes y el lugar de pertenencia institucional que generan. Es la institución la que produce reconocimiento de la tarea docente, la que posibilita socialización del trabajo cotidiano y hace comunes problemáticas que podrían parecer individuales.

\section{Palabras finales}

Este trabajo comienza con una pregunta, la formación permanente como acople o desacople en las instituciones escolares. Es un interrogante que en las experiencias presentadas, desde las concepciones docentes, tiene una respuesta singular. Lo primero que habría que decir es que nos encontramos ante un escenario educativo donde coexisten acoples y desacoples, donde las problemáticas comunes a todos los docentes, en cada establecimiento escolar emergen de un modo propio que precisa de una respuesta propia.

Con respecto al lugar de la formación permanente en las instituciones, puede observarse que cuando no lo hay, los docentes salen al encuentro de otros espacios en donde se puedan construir procesos de autoformación. Consideramos que este es un desacople institucional, en tanto la institución queda al margen: al margen de la formación, al margen de la producción, al margen del encuentro.

Cuando la institución queda al margen, pareciera que no hay intermediaciones entre las exigencias de las políticas educativas y el trabajo cotidiano. Allí donde la escuela debería pensar modos de traducción de esas exigencias 
en sus cotidianos, hay un lugar difuso, un desacople, tanto con las políticas educativas como con los docentes. Falta un interlocutor. Ante esto, nos preguntamos ¿Cómo poner en marcha espacios colectivos de pensamiento dentro de la escuela?

Lo dicho sobre la primer experiencia junto con la segunda experiencia presentada, demuestra, a nuestro entender, que el trabajo docente es colectivo, que precisa de la socialización y producción institucional y que en función de ello, es capaz de donar tiempo no reconocido en los contratos laborales. El trabajo docente precisa de sentido, del sentido político que tiene todo acto de educar.

\section{Fuentes}

- $\quad$ Ley Federal de Educación № 24.195

- $\quad$ Ley de Educación Superior № 24.521

- Ley de Educación Nacional № 26.206

- Resolución 36/94 CFCyE

- Resolución 52/96 CFCyE

- Resolución 63/97 CFCyE

- Resolución 135/00 CFCyE

- Resolución 144/00 CFCyE

- Resolución 144/00 CFCyE

- Resolución 152/00 CFCyE

- Resolución 167/01 CFCyE

- Resolución 173/02 CFCyE

- Resolución N² 251/05 CFCyE

- Registro $N^{\circ}$ 2. Entrevista con B. 18 de octubre del 2007.

- Registro No 1. Entrevista con G. 16 de mayo del 2007

- Registro № 2. Entrevista con G. 11 de octubre del 2007.

- Registro № 1. Entrevista con B. 13 de mayo del 2007.

- Registro No 1. Entrevista con P. 8 de junio de 2007.

- Registro № 3. Entrevista con S. 10 de diciembre del 2009.

- Registro № 1. Entrevista colectiva. 09 de Agosto del 2008.

\section{Notas}

(1) Este apartado contiene algunos fragmentos de reflexiones expuestas en otros artículos. En el presente, solo auspician de introducción a la temática que aquí nos ocupa, siendo la misma, elaboración original.

\section{Referencias Bibliográficas}

- $\quad$ Achilli, E.L. (2005). Investigar en antropología social. Los desafíos de transmitir un oficio. Rosario. Laborde Editor. 
- $\quad$ Agamben, G. (1998). El poder soberano y la nuda vida. Homo Sacer I. España: Ed. Pre-textos.

- Auge, M. (2001). No lugares y Espacio Público. En: Quaderns d'arquitectura i urbanisme, № 231. Recuperado en Febrero del 2009 de: http://www.farq.edu.uy/estructura/talleres/ perdomo/pagina\%2052.htm

- $\quad$ Bauman, Z. (1994). Pensando sociológicamente. Bs. As.: Nueva Visión.

- Gramsci, A. (1975).Cuadernos de la cárcel. Tomo III. Edición Crítica del Instituto Gramsci a cargo de Valentino Gerratana. En: http://books.google.com.ar. Consultado en mayo 2009.

- Mendizábal, N. (2007). Los componentes del diseño flexible en la investigación cualitativa. En Vasilachis I. (coord.) Estrategias de investigación cualitativa. Buenos Aires: Gedisa.

- Nicastro, S. (2005) Revisitar la mirada sobre la escuela. Exploraciones acerca de lo ya sabido. Rosario: HomoSapiens. 